\title{
A New Subclass of $k$-Janowski Type Functions Associated with Ruscheweyh Derivative
}

\author{
Shahid Mahmood, ${ }^{1}$ Sarfraz Nawaz Malik, ${ }^{2}$ Saima Mustafa, ${ }^{3}$ and S. M. Jawwad Riaz ${ }^{2}$ \\ ${ }^{1}$ Department of Mechanical Engineering, Sarhad University of Science \& IT, Landi Akhun Ahmad, Hayatabad Link, \\ Ring Road, Peshawar, Pakistan \\ ${ }^{2}$ Department of Mathematics, COMSATS Institute of Information Technology, Wah Cantt, Pakistan \\ ${ }^{3}$ Department of Statistics \& Mathematics, PMAS-Arid Agriculture University, Rawalpindi, Pakistan
}

Correspondence should be addressed to Shahid Mahmood; shahidmahmood757@gmail.com

Received 25 February 2017; Accepted 13 April 2017; Published 3 May 2017

Academic Editor: John R. Akeroyd

Copyright ( 2017 Shahid Mahmood et al. This is an open access article distributed under the Creative Commons Attribution License, which permits unrestricted use, distribution, and reproduction in any medium, provided the original work is properly cited.

We introduce and investigate a new subclass $\mathscr{V} \mathscr{D}_{k}(A, B, b, \delta)$ of analytic functions using Ruscheweyh derivative. We derive the coefficient inequalities and other interesting properties and characteristics for functions belonging to the general class, which we have introduced and studied in this article. We also observe that this class is preserved under the Bernardi integral transform.

\section{Introduction}

Let $\mathscr{A}$ denote the class of functions $f(z)$ of the form

$$
f(z)=z+\sum_{n=2}^{\infty} a_{n} z^{n}
$$

which are analytic in the unit disc $\mathfrak{D}=\{z:|z|<1\}$. Also let $\mathcal{S}^{*}(\beta)$ and $\mathscr{C}(\beta)$ denote the well-known classes of starlike and convex functions of order $\beta$, respectively. For details, see [1]. For any two analytic functions $f(z)$ and $g(z)$ with

$$
\begin{aligned}
& f(z)=z+\sum_{n=2}^{\infty} a_{n} z^{n}, \\
& g(z)=z+\sum_{n=2}^{\infty} b_{n} z^{n},
\end{aligned}
$$

for $z \in \mathfrak{D}$,

their convolution (Hadamard product) is given by

$$
(f * g)(z)=z+\sum_{n=2}^{\infty} a_{n} b_{n} z^{n}, \quad \text { for } z \in \mathfrak{D} .
$$

In 1975, using the concept of convolution, Ruscheweyh [2] introduced a linear operator $D^{\delta}: \mathscr{A} \rightarrow \mathscr{A}$ defined by

$$
D^{\delta} f(z)=\frac{z}{(1-z)^{\delta+1}} * f(z)=z+\sum_{n=2}^{\infty} \varphi_{n}(\delta) a_{n} z^{n}
$$

$$
(\delta>-1) \text {, }
$$

with

$$
\varphi_{n}(\delta)=\frac{(\delta+1)_{n-1}}{(n-1) !},
$$

where $(\rho)_{n}$ is a Pochhammer symbol given as

$$
(\rho)_{n}= \begin{cases}1, & n=0, \\ \rho(\rho+1)(\rho+2) \cdots(\rho+n-1), & n \in \mathbb{N} .\end{cases}
$$

It is obvious that $D^{0} f(z)=f(z), D^{1} f(z)=z f^{\prime}(z)$ and

$$
D^{n} f(z)=\frac{z\left(z^{n-1} f(z)\right)^{(n)}}{n !},
$$

$$
\forall \delta=n \in \mathbb{N}_{0}=\{0,1,2, \ldots\} .
$$


The following identity can easily be established:

$$
(\delta+1) D^{\delta+1} f(z)=\delta D^{\delta} f(z)+z\left(D^{\delta} f(z)\right)^{\prime} .
$$

The operator $D^{\delta} f(z)$ is called the Ruscheweyh derivative of $f(z)$; see [2].

Suppose also that, for $k \geq 0$, the classes $k-\mathscr{C V}$ and $k-\mathcal{S T}$ denote the well-known classes of $k$-uniformly convex and $k$ starlike functions, respectively. These classes were introduced by Kanas and Wisniowska [3, 4]. For some details see [3-5].
Consider the domain

$$
\Omega_{k}=\left\{u+i v ; u>k \sqrt{(u-1)^{2}+v^{2}}\right\} .
$$

For fixed $k, \Omega_{k}$ represents the conic region bounded successively by the imaginary axis $(k=0)$, the right branch of a hyperbola $(0<k<1)$, a parabola $(k=1)$, and an ellipse $(k>1)$. This domain was studied by Kanas [3-5]. The function $p_{k}$, with $p_{k}(0)=1, p_{k}^{\prime}(0)>0$, plays the role of extremal and is given by

$$
p_{k}(z)= \begin{cases}\frac{1+z}{1-z}, & k=0, \\ 1+\frac{2}{\pi^{2}}\left(\log \frac{1+\sqrt{z}}{1-\sqrt{z}}\right)^{2}, & k=1, \\ 1+\frac{2}{1-k^{2}} \sinh ^{2}\left[\left(\frac{2}{\pi} \arccos k\right) \operatorname{arctanh} \sqrt{z}\right], & 0<k<1, \\ 1+\frac{1}{k^{2}-1} \sin \left[\frac{\pi}{2 R(t)} \int_{0}^{u(z) / \sqrt{t}} \frac{1}{\sqrt{1-x^{2}} \sqrt{1-(t x)^{2}}} \mathrm{~d} x\right]+\frac{1}{k^{2}-1}, & k>1,\end{cases}
$$

where $u(z)=(z-\sqrt{t}) /(1-\sqrt{t z}), t \in(0,1), \quad z \in \mathfrak{D}$, and $t$ is chosen such that $k=\cosh \left(\pi R^{\prime}(t) / 4 R(t)\right)$, with $R(t)$ being Legendre's complete elliptic integral of the first kind and $R^{\prime}(t)$ being complementary integral of $R(t)$ (see $[3,4,6-8]$ ). Let $\mathscr{P}_{k}$ denote the class of all those functions $p(z)$ which are analytic in $\mathfrak{D}$ with $p(0)=1$ and $p(z) \prec p_{k}(z)$ for $z \in \mathfrak{D}$. Clearly it can be seen that $\mathscr{P}_{k} \subset \mathscr{P}$ where $\mathscr{P}$ is the class of functions with positive real part (see $[1,9])$. More precisely

$$
\mathscr{P}_{k} \subset \mathscr{P}\left(\frac{k}{1+k}\right) \subset \mathscr{P},
$$

and, for $f(z) \in \mathscr{P}_{k}$, we have

$$
|\arg p(z)| \leq \frac{\lambda \pi}{2},
$$

where

$$
\lambda=\frac{2}{\pi} \arctan \left(\frac{1}{k}\right)
$$

So we can write $p(z)=h^{\lambda}(z), h(z) \in \mathscr{P}$.

Definition 1 (see [10]). A function $p(z)$ is said to be in the class $\mathscr{P}_{k}[A, B]$, if and only if

$$
p(z) \prec p_{k}[A, B ; z]=\frac{(A+1) p_{k}(z)-(A-1)}{(B+1) p_{k}(z)-(B-1)},
$$

where $p_{k}(z)$ is defined by $(10)$ and $-1 \leq B<A \leq 1$. Geometrically, the function $p(z) \in \mathscr{P}_{k}[A, B]$ takes all values from the domain $\Omega_{k}[A, B],-1 \leq B<A \leq 1, k \geq 0$ which is defined as

$$
\begin{gathered}
\Omega_{k}[A, B]=\left\{w: \operatorname{Re}\left(\frac{(B-1) w(z)-(A-1)}{(B+1) w(z)-(A+1)}\right)\right. \\
\left.>k\left|\frac{(B-1) w(z)-(A-1)}{(B+1) w(z)-(A+1)}-1\right|\right\}
\end{gathered}
$$

or equivalently

$$
\begin{gathered}
\Omega_{k}[A, B]=\left\{u+i v:\left[\left(B^{2}-1\right)\left(u^{2}+v^{2}\right)-2(A B-1)\right.\right. \\
\left.\cdot u+\left(A^{2}-1\right)\right]^{2}>k^{2}\left[\left(-2(B+1)\left(u^{2}+v^{2}\right)\right.\right. \\
\left.\left.+2(A+B+2) u-2(A+1))^{2}+4(A-B)^{2} v^{2}\right]\right\} .
\end{gathered}
$$

The domain $\Omega_{k}[A, B]$ retains the conic domain $\Omega_{k}$ inside the circular region defined by $\Omega_{k}[A, B]$. The impact of $\Omega_{k}[A, B]$ on the conic domain $\Omega_{k}$ changes the original shape of the conic regions. The ends of hyperbola and parabola get closer to each other but never meet anywhere and the ellipse gets the shape of oval. When $A \rightarrow 1, B \rightarrow-1$, the radius of the circular disk defined by $\Omega[A, B]$ tends to infinity, consequently the arms of hyperbola and parabola expand, and the oval turns into ellipse.

With the help of the above Ruscheweyh derivative, we now define the following class.

Definition 2. A function $f(z) \in \mathscr{A}$ of the form (1) is in the class $\mathscr{V} \mathscr{D}_{k}(A, B, b, \delta)$ if and only if 


$$
\begin{gathered}
\mathfrak{R e}\left(\frac{(B-1)\left(1-2 / b+(2 / b)\left(D^{\delta+1} f(z) / D^{\delta} f(z)\right)\right)-(A-1)}{(B+1)\left(1-2 / b+(2 / b)\left(D^{\delta+1} f(z) / D^{\delta} f(z)\right)\right)-(A+1)}\right) \\
>k\left|\frac{(B-1)\left(1-2 / b+(2 / b)\left(D^{\delta+1} f(z) / D^{\delta} f(z)\right)\right)-(A-1)}{(B+1)\left(1-2 / b+(2 / b)\left(D^{\delta+1} f(z) / D^{\delta} f(z)\right)\right)-(A+1)}-1\right|,
\end{gathered}
$$

or equivalently,

$$
\left(1-\frac{2}{b}+\frac{2}{b} \frac{D^{\delta+1} f(z)}{D^{\delta} f(z)}\right) \prec p_{k}[A, B ; z], \quad z \in \mathfrak{D},
$$

with $p_{k}[A, B ; z]$ being given by (14), $k \geq 0,-1 \leq B<A \leq 1$, $\delta>-1$, and $b \in \mathbb{C}-\{0\}$.

For different permissible choices of parameters, we obtain several known as well as new subclasses of the class $\mathscr{A}$ of analytic functions as special cases; for example,

(i) For $\delta=0$ and $b=2$, we obtain $\mathscr{V} \mathscr{D}_{k}(A, B, 2,0)=$ $k-\mathcal{S} \mathscr{T}[A, B]$, and for $b=1$, we have the class $k-\mathcal{U} \mathscr{C} \mathscr{V}[A, B]$. These classes are recently introduced and studied by [10].

(ii) $\mathscr{V} \mathscr{D}_{k}(1,-1,2,0)=k-S T, \mathscr{V} \mathscr{D}_{k}(1,-1,1,0)=k-$ $\mathcal{U} \mathscr{C} \mathscr{V}$, the well-known classes of $k$-uniformly convex and $k$-starlike functions, respectively, introduced by Kanas and Wisniowska $[3,4]$.

(iii) $\mathscr{V} \mathscr{D}_{k}(1-2 \alpha,-1,2,0)=\delta \mathscr{D}(k, \alpha), \mathscr{V} \mathscr{D}_{k}(1-2 \alpha$, $-1,1,0)=\mathscr{K} \mathscr{D}(k, \alpha)$, the classes, introduced by Shams et al. in [11].

(iv) $\mathscr{V} \mathscr{D}_{0}(A, B, 2,0)=\mathcal{S}^{*}[A, B], \mathscr{V} \mathscr{D}_{0}(A, B, 1,0)=$ $\mathscr{C}[A, B]$, the well-known classes of Janowski starlike and Janowski convex functions, respectively, introduced by Janowski [12].

Throughout this paper, we assume that $k \geq 0, \delta>-1$, $-1 \leq B<A \leq 1$, and $b \in \mathbb{C}-\{0\}$ unless otherwise stated.

\section{Preliminary Results}

We need the following lemmas to obtain our results.

Lemma 3 (see [5]). Let $\sigma, \lambda$ with any complex numbers with $\lambda \neq 0$ and $0 \leq \gamma \leq \mathfrak{R e}(\lambda k /(k+1)+\sigma)$. If $\phi(z)$ is analytic in $\mathfrak{D}$ with $\phi(0)=1$ and satisfies

$$
\left(\phi(z)+\frac{z \phi^{\prime}(z)}{\lambda \phi(z)+\sigma}\right) \prec p_{k, \gamma}(z)
$$

and $\varphi_{k, \gamma}(z)$ is an analytic solution of

$$
\varphi_{k, \gamma}(z)+\frac{z \varphi_{k, \gamma}^{\prime}(z)}{\lambda \varphi_{k, \gamma}(z)+\sigma}=p_{k, \gamma}(z),
$$

then $\varphi_{k, \gamma}(z)$ is univalent,

$$
\phi(z) \prec \varphi_{k, \gamma}(z) \prec p_{k, \gamma}(z)
$$

and $\varphi_{k, \gamma}(z)$ is the best dominant of (19), where $\varphi_{k, \gamma}(z)$ is given as

$$
\begin{aligned}
& \varphi_{k, \gamma}(z) \\
& \quad=\left\{\left[\left(\int_{0}^{1} \exp \int_{t}^{t z} \frac{p_{k, \gamma}(u)-1}{u} \mathrm{~d} u\right) \mathrm{d} t\right]^{-1}+\frac{\sigma}{\lambda}\right\} .
\end{aligned}
$$

Lemma 4 (see [10]). Let $p(z)=1+\sum_{n=1}^{\infty} c_{n} z^{n} \in k-\mathscr{P}[A, B]$. Then

$$
\left|c_{n}\right| \leq\left|Q_{A B}\right|
$$

where

$$
\begin{aligned}
&\left|Q_{A B}\right|= \frac{(A-B)\left|Q_{k}\right|}{2}, \\
& Q_{k}= \begin{cases}\frac{8\left(\cos ^{-1} k\right)^{2}}{\pi^{2}\left(1-k^{2}\right)}, & 0 \leq k<1, \\
\frac{8}{\pi^{2}}, & k=1, \\
\frac{\pi^{2}}{4 \sqrt{t}\left(k^{2}-1\right) R^{2}(t)(1+t)}, & k>1 .\end{cases}
\end{aligned}
$$

Lemma 5 (see [13]). Let $p(z)=1+\sum_{n=1}^{\infty} p_{n} z^{n} \prec F(z)=1+$ $\sum_{n=1}^{\infty} d_{n} z^{n}$ in $\mathfrak{D}$. If $F(z)$ is univalent in $\mathfrak{D}$ and $F(\mathfrak{D})$ is convex, then

$$
\left|p_{n}\right| \leq\left|d_{1}\right|, \quad n \geq 1 \text {. }
$$

Lemma 6 (see $[14])$. Let $\beta_{0}>0, \beta_{0}+\gamma_{0}>0$ and $\alpha \in\left[\alpha_{0}, 1\right)$, where

$$
\alpha_{0}=\max \left\{\frac{\beta_{0}-\gamma_{0}-1}{2 \beta_{0}}, \frac{-\gamma_{0}}{\beta_{0}}\right\} .
$$

Let $h(z) \in \mathscr{A}$ in $\mathfrak{D}$ with $h(0)=1$ and let

$$
\left\{h(z)+\frac{z h^{\prime}(z)}{\beta_{0} h(z)+\gamma_{0}}\right\} \in \mathscr{P}(\alpha), \quad 0 \leq \alpha<1 .
$$

Then

Re $h(z)$

$$
>\left[\frac{\beta_{0}+\gamma_{0}}{\beta_{0}\left[{ }_{2} F_{1}\left[2 \beta_{0}(1-\alpha), 1, \beta_{0}+\gamma_{0}+1,1 / 2\right]\right]}\right.
$$

$$
\left.-\frac{\gamma_{0}}{\beta_{0}}\right]
$$


and the bound in (29) is sharp, the extremal functions being

$$
h_{0}=\frac{1}{\beta_{0} g_{0}(z)}-\frac{\gamma_{0}}{\beta_{0}},
$$

with

$$
g_{0}(z)=\int_{0}^{1}\left[\frac{1-z}{1-t z}\right]^{2 \beta_{0}(1-\alpha)} t^{\beta_{0}+\gamma_{0}-1} \mathrm{~d} t
$$

\section{Main Results}

Theorem 7. A function $f(z) \in \mathscr{A}$ and of the form (1) is in the class $\mathscr{V} \mathscr{D}_{k}(A, B, b, \delta)$, if it satisfies the condition

$$
\begin{aligned}
\sum_{n=2}^{\infty} & \left\{\frac{4(n-1)(k+1)}{\delta+1}\right. \\
+ & \left.\left|\frac{2(B+1)(\delta+n)}{\delta+1}+b(B-A)-2(B+1)\right|\right\} \\
\cdot & \varphi_{n}(\delta)\left|a_{n}\right|<|b||B-A| .
\end{aligned}
$$

Proof. Let we note that

$$
\begin{aligned}
& \left|\frac{(B-1)\left(1-2 / b+(2 / b)\left(D^{\delta+1} f(z) / D^{\delta} f(z)\right)\right)-(A-1)}{(B+1)\left(1-2 / b+(2 / b)\left(D^{\delta+1} f(z) / D^{\delta} f(z)\right)\right)-(A+1)}-1\right| \\
& \quad=4\left|\frac{D^{\delta+1} f(z)-D^{\delta} f(z)}{2(B+1) D^{\delta+1} f(z)+(b(B-A)-2(B+1)) D^{\delta} f(z)}\right| \\
& \quad=\left|\frac{\sum_{n=2}^{\infty}\{4(n-1) /(\delta+1)\} \varphi_{n}(\delta) a_{n} z^{n}}{b(B-A) z+\sum_{n=2}^{\infty}\{2(B+1)(\delta+n) /(\delta+1)+b(B-A)-2(B+1)\} \varphi_{n}(\delta) a_{n} z^{n}}\right| \\
& \quad \leq \frac{\sum_{n=2}^{\infty}\{4(n-1) /(\delta+1)\} \varphi_{n}(\delta)\left|a_{n}\right|}{|b||B-A|-\sum_{n=2}^{\infty}|2(B+1)(\delta+n) /(\delta+1)+b(B-A)-2(B+1)| \varphi_{n}(\delta)\left|a_{n}\right|}
\end{aligned}
$$

because from (32) it follows that

$$
\begin{aligned}
& \{|b||B-A| \\
& -\sum_{n=2}^{\infty}\left|\frac{2(B+1)(\delta+n)}{\delta+1}+b(B-A)-2(B+1)\right| \\
& \qquad\left|\frac{(B-1)\left(1-2 / b+(2 / b)\left(D^{\delta+1} f(z) / D^{\delta} f(z)\right)\right)-(A-1)}{(B+1)\left(1-2 / b+(2 / b)\left(D^{\delta+1} f(z) / D^{\delta} f(z)\right)\right)-(A+1)}-1\right| \\
& \\
& \quad-\operatorname{Re}\left(\frac{(B-1)\left(1-2 / b+(2 / b)\left(D^{\delta+1} f(z) / D^{\delta} f(z)\right)\right)-(A-1)}{(B+1)\left(1-2 / b+(2 / b)\left(D^{\delta+1} f(z) / D^{\delta} f(z)\right)\right)-(A+1)}\right)<1 .
\end{aligned}
$$

From (33), we have

$$
\begin{aligned}
k\left|\frac{(B-1)\left(1-2 / b+(2 / b)\left(D^{\delta+1} f(z) / D^{\delta} f(z)\right)\right)-(A-1)}{(B+1)\left(1-2 / b+(2 / b)\left(D^{\delta+1} f(z) / D^{\delta} f(z)\right)\right)-(A+1)}-1\right| \\
\quad-\operatorname{Re}\left(\frac{(B-1)\left(1-2 / b+(2 / b)\left(D^{\delta+1} f(z) / D^{\delta} f(z)\right)\right)-(A-1)}{(B+1)\left(1-2 / b+(2 / b)\left(D^{\delta+1} f(z) / D^{\delta} f(z)\right)\right)-(A+1)}\right)
\end{aligned}
$$




$$
\begin{aligned}
& \leq(k+1)\left|\frac{(B-1)\left(1-2 / b+(2 / b)\left(D^{\delta+1} f(z) / D^{\delta} f(z)\right)\right)-(A-1)}{(B+1)\left(1-2 / b+(2 / b)\left(D^{\delta+1} f(z) / D^{\delta} f(z)\right)\right)-(A+1)}-1\right| \\
& \leq \frac{\sum_{n=2}^{\infty}\{4(n-1)(k+1) /(\delta+1)\} \varphi_{n}(\delta)\left|a_{n}\right|}{|b||B-A|-\sum_{n=2}^{\infty}|2(B+1)(\delta+n) /(\delta+1)+b(B-A)-2(B+1)| \varphi_{n}(\delta)\left|a_{n}\right|} .
\end{aligned}
$$

The last expression is bounded above by 1 if

$$
\begin{aligned}
& \sum_{n=2}^{\infty}\left\{\frac{4(n-1)(k+1)}{\delta+1}\right. \\
& \left.+\left|\frac{2(B+1)(\delta+n)}{\delta+1}+b(B-A)-2(B+1)\right|\right\} \\
& \cdot \varphi_{n}(\delta)\left|a_{n}\right|<|b||B-A|,
\end{aligned}
$$

and this completes the proof.

When we put $\delta=0$ and $b=2$ in the above theorem, we obtain the following known result, proved by Noor and Malik in $[10]$.

Corollary 8. A function $f(z) \in \mathscr{A}$ and of the form (1) is in the class $k-\mathcal{S} \mathscr{T}[A, B]$, if it satisfies the condition

$$
\begin{aligned}
& \sum_{n=2}^{\infty}\{2(n-1)(k+1)+|(B+1) n-(1+A)|\} \varphi_{n}(\delta)\left|a_{n}\right| \\
& \quad<|B-A| .
\end{aligned}
$$

For $A=1-2 \alpha, B=-1$ with $0 \leq \alpha<1, \delta=0$, and $b=2$, Theorem 7 reduces to the following known result, proved by Shams et al. [11].

Corollary 9. A function $f(z) \in \mathscr{A}$ and of the form (1) is in the class $\mathcal{S} \mathscr{D}(k, \alpha)$, if it satisfies the condition

$$
\sum_{n=2}^{\infty}\{n(k+1)-(k+\alpha)\}\left|a_{n}\right|<1-\alpha .
$$

Theorem 10. Let $f(z) \in \mathscr{V} \mathscr{D}_{k}(A, B, b, \delta)$. Then

$$
\left|a_{n}\right| \leq \frac{(\sigma)_{n-1}}{(n-1) ! \varphi_{n}(\delta)}, \text { for } n \geq 2,
$$

where

$$
\sigma=\frac{|b|\left|Q_{A B}\right|(\delta+1)}{2},
$$

where $\left|Q_{A B}\right|$ and $\varphi_{n}(\delta)$ are given by (24) and (5).

Proof. Set

$$
1-\frac{2}{b}+\frac{2}{b} \frac{D^{\delta+1} f(z)}{D^{\delta} f(z)}=p(z)
$$

so that $p(z) \in \mathscr{P}_{k}[A, B]$. Let $p(z)=1+\sum_{n=1}^{\infty} c_{n} z^{n}$. Then (42) can be written as

$$
2\left(D^{\delta+1} f(z)-D^{\delta} f(z)\right)=b D^{\delta} f(z) \sum_{n=1}^{\infty} c_{n} z^{n},
$$

which implies that

$$
\begin{aligned}
& \frac{2 \varphi_{n}(\delta)(n-1) a_{n}}{(\delta+1)} \\
& \quad=b\left(c_{n-1}+\varphi_{2}(\delta) a_{2} c_{n-2}+\cdots+\varphi_{n-1}(\delta) a_{n-1} c_{1}\right) .
\end{aligned}
$$

Using the coefficient estimates $\left|c_{n}\right| \leq\left|Q_{A B}\right|$ for the class $\mathscr{P}_{k}[A, B]$ (see $\left.[10]\right)$, we obtain

$$
\begin{aligned}
\left|a_{n}\right| & \leq \frac{|b|\left|Q_{A B}\right|(\delta+1)}{2(n-1) \varphi_{n}(\delta)}\left(1+\varphi_{2}(\delta)\left|a_{2}\right|+\cdots\right. \\
& \left.+\varphi_{n-1}(\delta)\left|a_{n-1}\right|\right) .
\end{aligned}
$$

For $n=2$,

$$
\left|a_{2}\right| \leq \frac{|b|\left|Q_{A B}\right|}{2}
$$

Therefore (40) holds for $n=2$. Assume that (40) is true for $n=m$ and consider

$$
\begin{aligned}
& \left|a_{m+1}\right| \leq \frac{|b|\left|Q_{A B}\right|(\delta+1)}{2 m \varphi_{m+1}(\delta)}\left(1+\varphi_{2}(\delta)\left|a_{2}\right|+\cdots\right. \\
& \left.+\varphi_{n-1}(\delta)\left|a_{m}\right|\right) \\
& \quad \leq \frac{|b|\left|Q_{A B}\right|(\delta+1)}{2 m \varphi_{m+1}(\delta)}\left\{+\frac{|b|\left|Q_{A B}\right|(\delta+1)}{2 !}(1\right. \\
& \left.+\frac{|b|\left|Q_{A B}\right|(\delta+1)}{2}\right)+\cdots+\frac{|b|\left|Q_{A B}\right|(\delta+1)}{(m-1) !} \\
& \left.\quad \prod_{j=1}^{m-2}\left(1+\frac{|b|\left|Q_{A B}\right|(\delta+1)}{2 j}\right)\right\} \\
& \quad=\frac{|b|\left|Q_{A B}\right|(\delta+1)}{2 m \varphi_{m+1}(\delta)} \prod_{j=1}^{m-1}\left(1+\frac{|b|\left|Q_{A B}\right|(\delta+1)}{2 j}\right) \\
& \quad=\frac{(\sigma)_{m}}{(m) ! \varphi_{m+1}(\delta)} .
\end{aligned}
$$

Therefore, the result is true for $n=m+1$. Using mathematical induction, (40) holds true for all $n \geq 2$. 
Corollary 11 (see [10]). If $f(z) \in \mathscr{V} \mathscr{D}_{k}(A, B, 2,0)=k-$ $\mathcal{S} \mathscr{T}[A, B]$, then

$$
\left|a_{n}\right| \leq \prod_{j=0}^{n-2} \frac{\left|Q_{k}(A-\beta)-2 j B\right|}{2(j+1)}, \text { for } n \geq 2,
$$

where $Q_{k}$ is defined by (8).

When $A=1, B=-1, b=2$, and $\delta=0$, we obtain the following coefficient inequality for the class $k-\mathcal{S T}$, introduced by Kanas and Wisniowska [4].

Corollary 12. If $f(z) \in k-\mathcal{S T}$, then

$$
\left|a_{n}\right| \leq \prod_{j=0}^{n-2} \frac{\left|Q_{k}+j\right|}{j+1}, \text { for } n \geq 2 .
$$

This result is sharp.

By taking the values $A=1-2 \alpha$ with $0 \leq \alpha<1, B=-1$, $b=2$, and $\delta=0$, we obtain the coefficient inequality of the class $\mathcal{S} \mathscr{D}(k, \alpha)$, introduced by Shams et al. [11].

Corollary 13. If $f(z) \in \mathcal{S} \mathscr{D}(k, \alpha)$, then

$$
\left|a_{n}\right| \leq \prod_{j=0}^{n-2} \frac{\left|Q_{k}(1-\alpha)+j\right|}{j+1}, \text { for } n \geq 2 .
$$

This result is sharp.

Theorem 14. For real $b>0$, let $f(z) \in \mathscr{V} \mathscr{D}_{k}(A, B, b, \delta+1)$. Then $f(z) \in \mathscr{V} \mathscr{D}_{k}(A, B, b+1, \delta)$ for $z \in \mathfrak{D}$.

Proof. Suppose $f(z) \in \mathscr{V} \mathscr{D}_{k}(A, B, b, \delta+1)$ and set

$$
p(z)=1-\frac{2}{b+1}+\frac{2}{b+1} \frac{D^{\delta+1} f(z)}{D^{\delta} f(z)},
$$

where $p(z)$ is analytic in $\mathfrak{D}$ with $p(0)=1$. Then simple computations, together with (51) and (8), yield

$$
\begin{aligned}
1-\frac{2}{b}+\frac{2}{b} \frac{D^{\delta+2} f(z)}{D^{\delta+1} f(z)}= & \left(1-\lambda_{1}\right) \\
& +\lambda_{1}\left[p(z)+\frac{\lambda_{2} z p^{\prime}(z)}{p(z)+\lambda_{3}}\right],
\end{aligned}
$$

with $\lambda_{1}=(\delta+1)(b+1) / b(\delta+2), \quad \lambda_{2}=2 /(\delta+1)(b+1), \quad \lambda_{3}=$ $2 /(b+1)-1$. Since $f(z) \in \mathscr{V} \mathscr{D}_{k}(A, B, b, \delta+1)$, it follows that

$$
\begin{aligned}
& {\left[\left(1-\lambda_{1}\right)+\lambda_{1}\left(p(z)+\frac{\lambda_{2} z p^{\prime}(z)}{p(z)+\lambda_{3}}\right)\right]=h_{0}(z)} \\
& \quad \in \mathscr{P}_{k}[A, B],
\end{aligned}
$$

or, equivalently,

$$
\begin{aligned}
p(z) & +\frac{z p^{\prime}(z)}{\left(1 / \lambda_{2}\right) p(z)+\lambda_{3} / \lambda_{2}} \\
= & \frac{1}{\lambda_{1}} h_{0}(z)+\left(1-\frac{1}{\lambda_{1}}\right) p_{0}(z), \quad\left(p_{0}(z)=1\right) .
\end{aligned}
$$

Since $h_{0}(z), p_{0}(z) \in \mathscr{P}_{k}[A, B]$ and $\mathscr{P}_{k}[A, B]$ is a convex set (see [10]), it follows that $\left(1 / \lambda_{1}\right) h_{0}(z)+\left(1-1 / \lambda_{1}\right) p_{0}(z)$, with $p_{0}(z)=1$, belong to $\mathscr{P}_{k}[A, B]$ in $\mathfrak{D}$ and hence $p(z)+$ $z p^{\prime}(z) /\left(\left(1 / \lambda_{2}\right) p(z)+\lambda_{3} / \lambda_{2}\right) \in \mathscr{P}_{k}[A, B]$. We now use the Lemma 3 with $\eta=1 / \lambda_{2}, \sigma=\lambda_{3} / \lambda_{2}$, and $\Re \mathfrak{R}(\eta k /(k+1)+\sigma)>$ 0 to obtain $p(z) \in \mathscr{P}_{k}[A, B]$ and hence $f(z) \in \mathscr{V} \mathscr{D}_{k}(A, B, b+$ $1, \delta)$. This complete the proof.

For a function $f(z) \in \mathscr{A}$, we consider the integral operator

$$
F(z)=I_{c}(f(z))=\frac{(c+1)}{z^{c}} \int_{0}^{z} t^{c-1} f(t) \mathrm{d} t, \quad c>-1 .
$$

The operator $I_{c}$ was introduced by Bernardi [15] for $c \in \mathbb{N}$. In particular, the operator $I_{1}$ was studied earlier by Libera [16] and Livingston [17].

Theorem 15. Let $f(z) \in V \mathscr{D}_{k}(A, B, b, \delta)$ and let $F(z)$ be defined by (55). Then $F(z) \in \mathscr{V}_{k}(A, B, b, \delta)$.

Proof. Let

$$
1-\frac{2}{b}+\frac{2}{b} \frac{D^{\delta+1}(F(z))}{D^{\delta}(F(z))}=p(z),
$$

where $p(z)$ is analytic in $\mathfrak{D}, p(0)=1$. Then using (8), we have

$$
\frac{z\left(D^{\delta}(F(z))\right)^{\prime}}{D^{\delta}(F(z))}=a p(z)-a+1,
$$

where $a=b(\delta+1) / 2$. Simple computation and use of (8), (55), and (56), we have

$$
\begin{aligned}
1- & \frac{2}{b}+\frac{2}{b} \frac{D^{\delta+1}(f(z))}{D^{\delta}(f(z))}=p(z)+\frac{z p^{\prime}(z)}{a p(z)-a+1+c} \\
& \prec p_{k}[A, B ; z] .
\end{aligned}
$$

Apply Lemma 3 with $\eta=a, \sigma=1-a+c$ and $\mathfrak{R e}(\eta k /(k+$ 1) $+\sigma)>0$ to obtain $p(z) \prec p_{k}[A, B ; z]$ and consequently $F(z) \in \mathscr{V} \mathscr{D}_{k}(A, B, b, \delta)$.

Corollary 16. Let $b>0, f(z) \in V \mathscr{D}_{k}(A, B, b, \delta) \subset \mathscr{V} \mathscr{D}_{\alpha_{1}}(b$, $\delta)$, and $\alpha_{1}=(2 k+1-A) /(2 k+1-B)$ and let $F(z)$ be defined by (55). Then $F(z) \in \mathscr{V} \mathscr{D}_{\beta}(b, \delta)$, where $c>-1$, and

$$
\begin{aligned}
\beta & =\left\{\frac{c+1}{a\left[{ }_{2} F_{1}\left(2 a\left(1-\alpha_{1}\right), 1 ; c+2 ; 1 / 2\right)\right]}\right. \\
& \left.-\frac{1+c-a}{a}\right\} .
\end{aligned}
$$

Proof. Proceeding as in Theorem 15, it follows from (58) that

$$
\begin{aligned}
1- & \frac{2}{b}+\frac{2}{b} \frac{D^{\delta+1}(f(z))}{D^{\delta}(f(z))}=p(z)+\frac{z p^{\prime}(z)}{a p(z)-a+1+c} \\
& \in \mathscr{P}\left(\alpha_{1}\right), \quad \alpha_{1}=\frac{2 k+1-A}{2 k+1-B} .
\end{aligned}
$$

Applying Lemma 6 , we obtain $p(z) \in \mathscr{P}(\beta)$, where $\beta$ is given by (59). This proves that $F(z) \in \mathscr{V} \mathscr{D}_{\beta}(b, \delta)$ in $\mathfrak{D}$. 
Theorem 17. If $f(z)$ is of the form (1) belonging to $\mathscr{V} \mathscr{D}_{k}(A$, $B, b, \delta)$ and $F(z)=z+\sum_{n=2}^{\infty} b_{n} z^{n}$, where $F(z)$ is the integral operator defined by (55), then

$$
\left|b_{n}\right| \leq \frac{(c+1)}{(c+n)} \frac{(\sigma)_{n-1}}{(n-1) ! \varphi_{n}(\delta)}, \text { for } n \geq 2 .
$$

Proof. From (55), we obtain

$$
(c+1) f(z)=c F(z)+z F^{\prime}(z) .
$$

Using the series for the functions $f(z)$ and $F(z)$, we obtain

$$
\begin{aligned}
& (1+c) z+\sum_{n=2}^{\infty}(1+c) a_{n} z^{n} \\
& =c z+\sum_{n=2}^{\infty} c b_{n} z^{n}+z+\sum_{n=2}^{\infty} n b_{n} z^{n}
\end{aligned}
$$

and thus

$$
(n+c) b_{n}=(1+c) a_{n}, \quad n \geq 2 .
$$

From the above we have

$$
\left|b_{n}\right| \leq \frac{(c+1)}{(c+n)}\left|a_{n}\right|, \quad n \geq 2 .
$$

Using the estimates from Theorem 10, we obtain the required result.

\section{Conflicts of Interest}

The authors declare that there are no conflicts of interest regarding the publication of this article.

\section{Acknowledgments}

The authors would also like to acknowledge Professor Dr. Salim ur Rehman, V.C. Sarhad University of Science \& IT, for providing excellent research and academic environment.

\section{References}

[1] A. W. Goodman, Univalent Functions, vol. I, II, Polygonal Publishing House, Washington, DC, USA, 1983.

[2] S. Ruscheweyh, "New criteria for univalent functions," Proceedings of the American Mathematical Society, vol. 49, pp. 109-115, 1975.

[3] S. Kanas and A. Wisniowska, "Conic regions and $k$-uniform convexity," Journal of Computational and Applied Mathematics, vol. 105, pp. 327-336, 1999.

[4] S. Kanas and A. Wisniowska, "Conic domains and starlike functions," Revue Roumaine de Mathématiques Pures et Appliquées, vol. 45 , no. 4 , pp. $647-657,2000$.

[5] S. Kanas, "Techniques of the differential subordination for domains bounded by conic sections," International Journal of Mathematics and Mathematical Sciences, vol. 38, pp. 2389-2400, 2003.
[6] M. Arif, S. Mahmood, J. Sokol, and J. Dziok, "New subclass of analytic functions in conical domain associated with a linear operator," Acta Mathematica Scientia, Series B: English Edition, vol. 36, no. 3, pp. 704-716, 2016.

[7] S. Mahmood and J. Sokół, "New subclass of analytic functions in conical domain associated with ruscheweyh $q$-differential operator," Results in Mathematics, 2016.

[8] W. Ul-Haq and S. Mahmood, "Certain properties of a class of close-to-convex functions related to conic domains," Abstract and Applied Analysis, vol. 2013, Article ID 847287, 6 pages, 2013.

[9] B. Pinchuk, "Functions of bounded boundary rotation," Israel Journal of Mathematics, vol. 10, pp. 6-16, 1971.

[10] K. I. Noor and S. N. Malik, "On coefficient inequalities of functions associated with conic domains," Computers \& Mathematics with Applications, vol. 62, pp. 2209-2217, 2011.

[11] S. Shams, S. R. Kulkarni, and J. M. Jahangiri, "Classes of uniformly starlike and convex functions," International Journal of Mathematics and Mathematical Sciences, vol. 2004, no. 55, pp. 2959-2961, 2004.

[12] W. Janowski, "Some extremal problems for certain families of analytic functions-I," Annales Polonici Mathematici, vol. 28, pp. 297-326, 1973.

[13] W. Rogosinski, "On the coefficients of subordinate functions," Proceedings of the London Mathematical Society, vol. 48, pp. 4882, 1943.

[14] S. S. Miller, "Differential inequalities and Carathéodory functions," Bulletin of the American Mathematical Society, vol. 81, pp. 79-81, 1975.

[15] S. D. Bernardi, "Convex and starlike univalent functions," Transactions of the American Mathematical Society, vol. 135, pp. 429-446, 1969 .

[16] R. J. Libera, "Some classes of regular univalent functions," Proceedings of the American Mathematical Society, vol. 16, pp. 755-758, 1965.

[17] A. E. Livingston, "On the radius of univalence of certain analytic functions," Proceedings of the American Mathematical Society, vol. 17, pp. 352-357, 1966. 


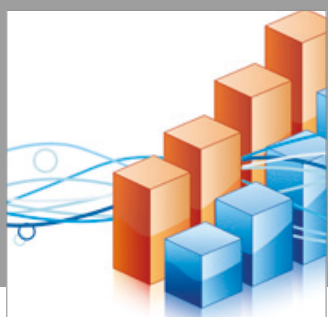

Advances in

Operations Research

vatersals

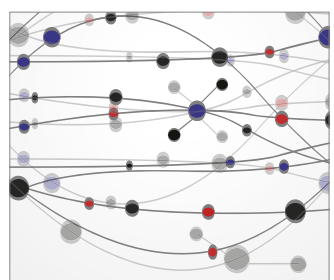

\section{The Scientific} World Journal
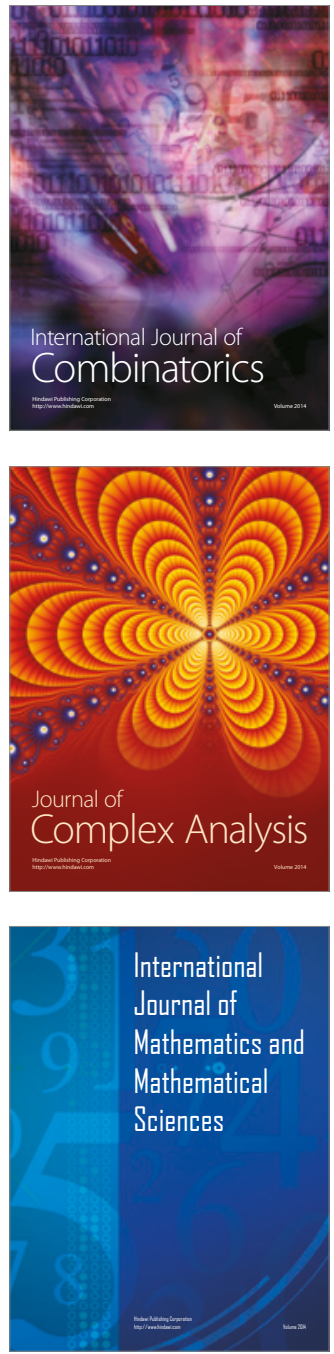
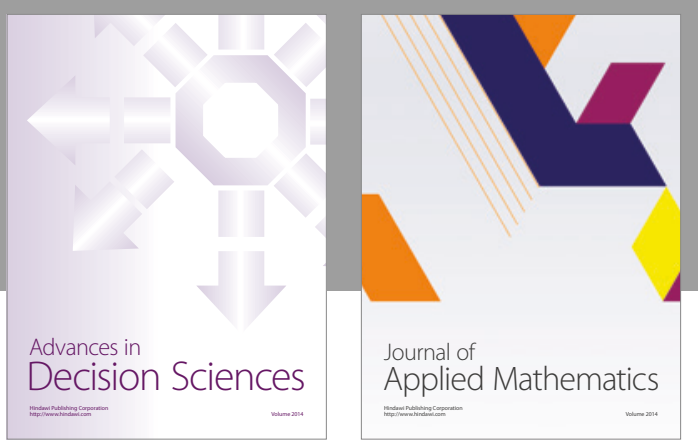

Algebra

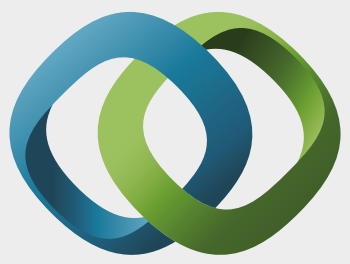

\section{Hindawi}

Submit your manuscripts at

https://www.hindawi.com
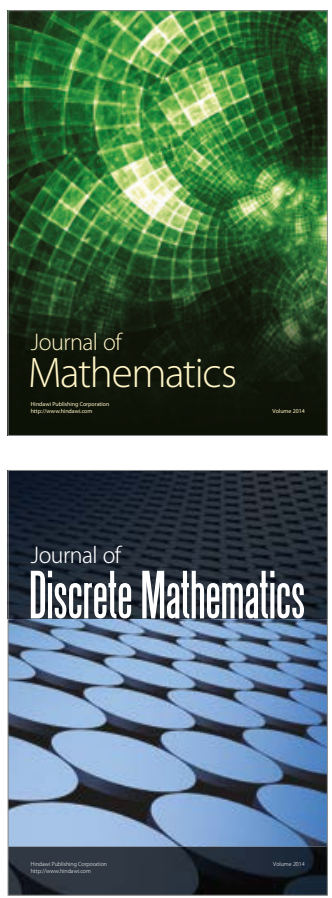

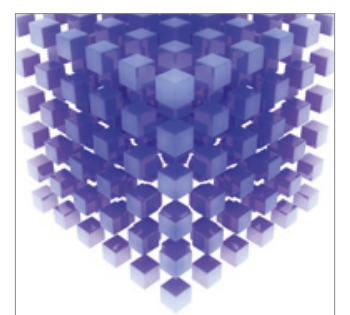

Mathematical Problems in Engineering
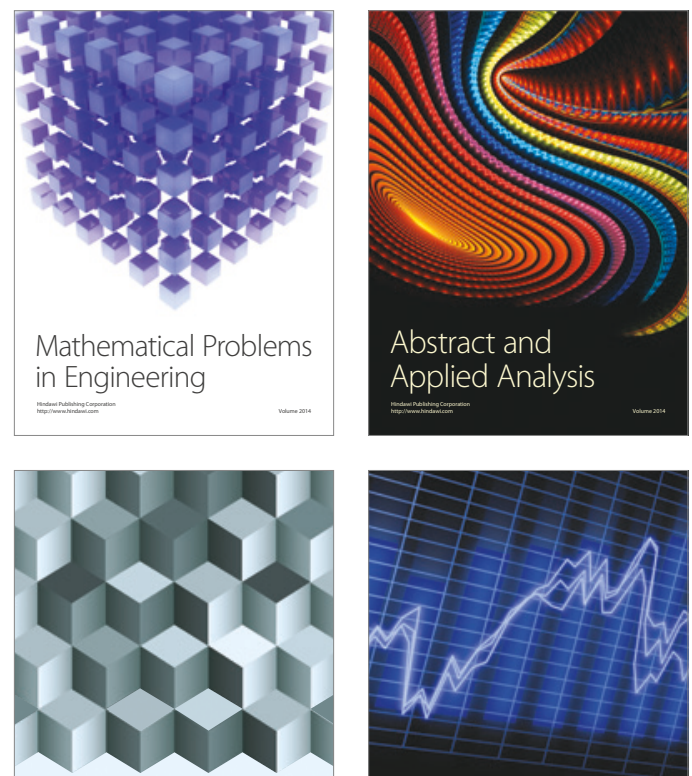

Journal of

Function Spaces

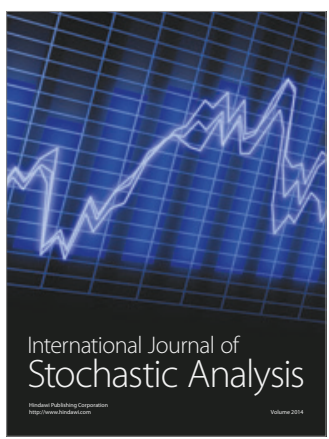

Probability and Statistics
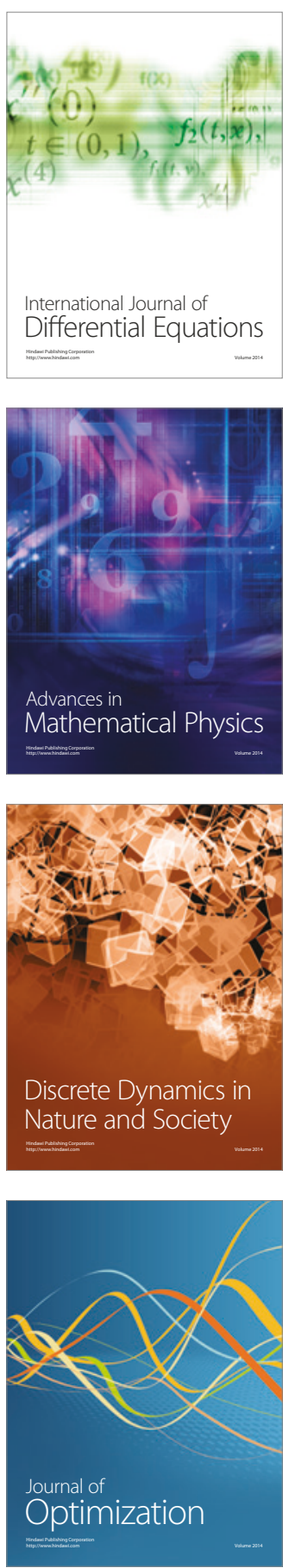